\title{
Clinical Trial Source Data
}

National Cancer Institute

\section{Source}

National Cancer Institute. Clinical Trial Source Data. NCI Thesaurus. Code C125442.

Information in original records and certified copies of original records of clinical findings, observations, or other activities in a clinical trial necessary for the reconstruction and evaluation of the trial. 